\title{
Dermatofibroma With Sebaceous Induction: Dermoscopic Clues to Improve Recognition
}

\author{
R. Hal Flowers ${ }^{1}$, Darren Guffey ${ }^{1}$, Jonathan C. Konopinski², \\ Michael A. Marchetti ${ }^{3}$, Barbara B. Wilson ${ }^{1}$
}

1 Department of Dermatology, University of Virginia Health System, Charlottesville, VA, USA

2 Department of Dermatopathology, University of Virginia Health System, Charlottesville, VA, USA

3 Dermatology Service, Department of Medicine, Memorial Sloan Kettering, New York, NY, USA

Key words: dermoscopy, dermatofibroma, sebaceous induction

Citation: Flowers RH, Guffey D, Konopinski JC, Marchetti MA, Wilson BB. Dermatofibroma with sebaceous induction: dermoscopic clues to improve recognition. Dermatol Pract Concept. 2019;9(4):315-317. DOI: https://doi.org/10.5826/dpc.0904a18

Accepted: May 25, 2019; Published: October 31, 2019

Copyright: $@ 2019$ Flowers et al. This is an open-access article distributed under the terms of the Creative Commons Attribution License, which permits unrestricted use, distribution, and reproduction in any medium, provided the original author and source are credited.

Funding: M.A.M. has support from a National Institutes of Health support grant to Memorial Sloan Kettering Cancer Center (P30 CA008748).

Competing interests: The authors have no conflicts of interest to disclose.

Authorship: All authors have contributed significantly to this publication.

Corresponding author: R. Hal Flowers, MD, 1221 Lee St., P.O. Box 800718, UVA Health System, Charlottesville, VA 22908-0718. Email: rf9rj@virginia.edu

\section{Introduction}

Dermatofibromas (DFs) can manifest as atrophic, depressed papules on the upper trunk and proximal upper extremities. Histologically, DFs may demonstrate sebaceous induction in the upper dermis, particularly if they occur on the shoulder [1]. On dermoscopy, these glands are readily visible as discrete pale yellow clods, reminiscent of so-called "popcorn-like" structures present in sebaceous hyperplasia. However, this finding is rarely cited as a dermoscopic feature in DFs. Here we detail a series of DFs presenting as atrophic papules with dermoscopic findings of yellow clods.

\section{Case Presentation}

We present 6 cases, descriptions of which follow: a 68-yearold woman with a 9- $\times 8$-mm atrophic DF on the right upper back (case 1: Figure 1, A-C); a 71-year-old man with an $8-x$ 5 -mm brown depressed DF on the right proximal forearm (case 2: Figure 2A); a 68-year-old man with a 1-cm pink- brown atrophic DF on the left upper arm (case 3: Figure 2B); a 56-year-old man with an atrophic reddish DF on the right upper extremity (case 4: Figure 2C); an 82-year-old man with an atrophic reddish papule on the upper back (case 5: Figure 2D); and a 55-year-old man with a pink plaque on the chest (case 6: Figure 2E).

\section{Discussion}

Histologically, the presence of sebaceous glands and sebaceous induction overlying DFs has been well documented. This occurs in at least $15 \%$ of samples, particularly in DFs on the shoulder [1]. Notably, the upper trunk is the most common site of clinically atrophic DFs. Sebaceous hyperplasia in this setting likely represents epidermal induction and may be mediated by fibroblast-released growth mediators [1].

The dermoscopic findings present in DFs with sebaceous induction have been very rarely described in the literature [1]. However, widespread appreciation of this dermoscopic feature in DFs appears low, as evidenced in their exclusion in 


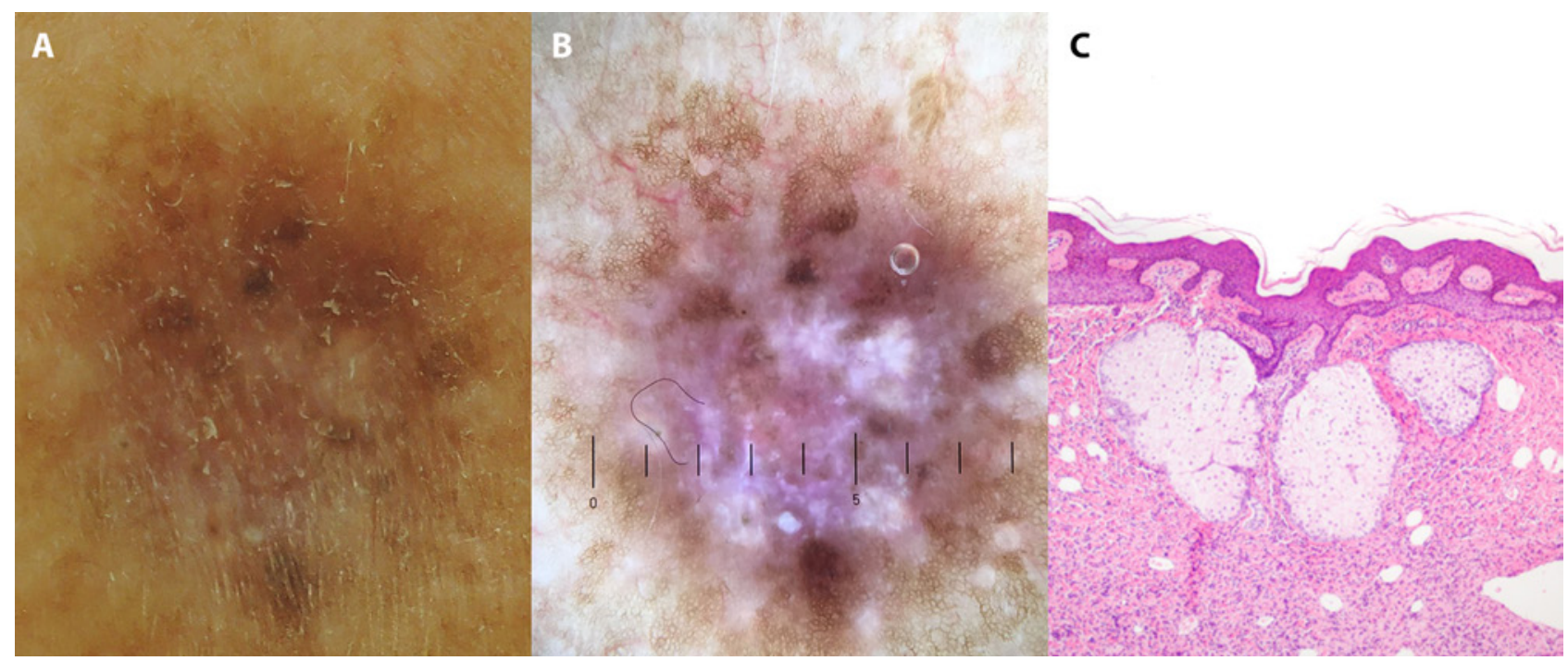

Figure 1. (A) Case 1 had a 9- $\times 8-\mathrm{mm}$ atrophic violaceous papule with several foci of dark brown pigmentation and inferiorly located whitish structures. (B) Dermoscopy of case 1 shows round white-yellow clods admixed with central scar-like patch and a peripheral pigment network. (C) Histological examination of case 1 shows a cellular dermal infiltrate composed of bland fibrohistiocytic cells. The epidermis is acanthotic, exhibiting a broadening and anastomosis of pale-staining rete ridges parallel to the skin surface. Notably, there is induction of sebaceous glands (H\&E, ×4). [Copyright: @2019 Flowers et al.]

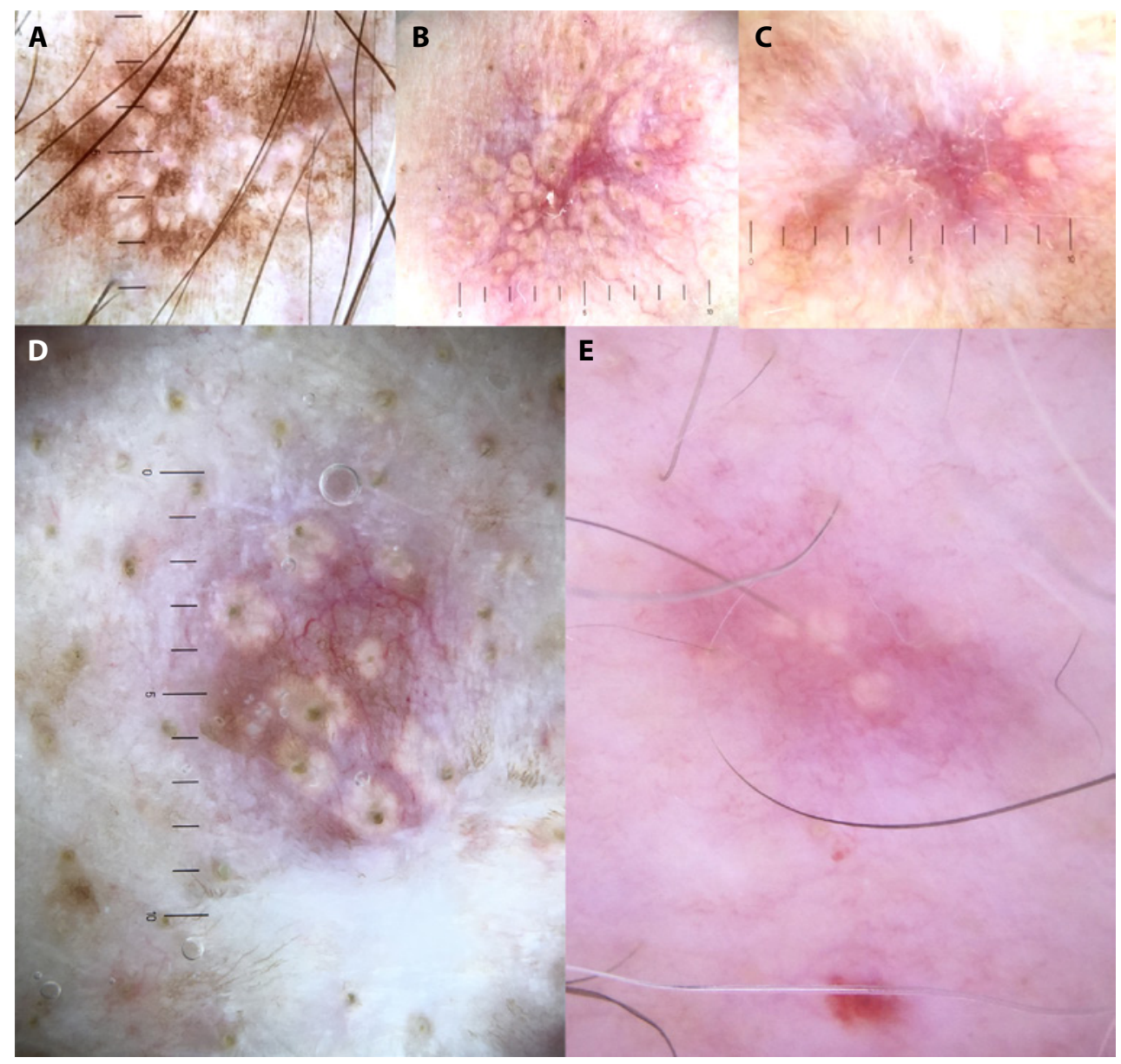

Figure 2. (A) Dermoscopy of case 2 shows a patchy peripheral pigment network and central white structureless area with multiple white-yellow clods. (B) Dermoscopy of case 3 shows a prominent vascularity with numerous yellow clods of varying sizes. (C) Dermoscopy of case 4 shows faint peripheral pigment network, central red patch, and a few admixed yellow clods. (D) Dermoscopy of case 5 shows background brownish homogeneous pigmentation with broad telangiectasias and scattered yellow clods with prominent follicular orifices. (E) Dermoscopy of case 6 shows background erythema with a few overlying central yellow clods. [Copyright: (C2019 Flowers et al.]

reported classifications of DF patterns. In these reports, yellowish globular structures on dermoscopy were histopathologically correlated with a prominent sebaceous component. Review of the literature reveals that previous dermoscopic descriptions of these structures exist, but that the correlation with sebaceous glands is not always made. For example, Karaarslan et al show a dermoscopic image of an atrophic DF and describe "multiple, small scar-like areas"; they include a histological image with prominent sebaceous glands, which appear to correspond to these "scar-like areas" [2]. 


\section{Conclusions}

We have observed that highly characteristic yellowish white discrete round clods are often present on dermoscopy of DFs which are clinically depressed or inverted (clinically atrophic DFs) on the upper torso, shoulder, and upper extremities. These yellow to yellow-white clods on dermoscopy correlate with sebaceous glands found in DFs. This dermoscopic finding may help the clinical recognition of a subset of atrophic DFs, which often lack the characteristic dimpling and palpability of typical DFs.

\section{References}

1. Monteagudo B, Figueroa-Silva O, Suárez-Amor O, Álvarez JC. Hallazgos clínicos, dermatoscópicos e histopatologicos de un dermatofibroma con inducción sebácea [in English, Spanish]. Actas Dermosifiliogr. 2017;108(9):874-876.

2. Karaarslan IK, Gencoglan G, Akalin T, Ozdemir F. Different dermoscopic faces of dermatofibromas. J Am Acad Dermatol. 2007;57(3):401-406. 